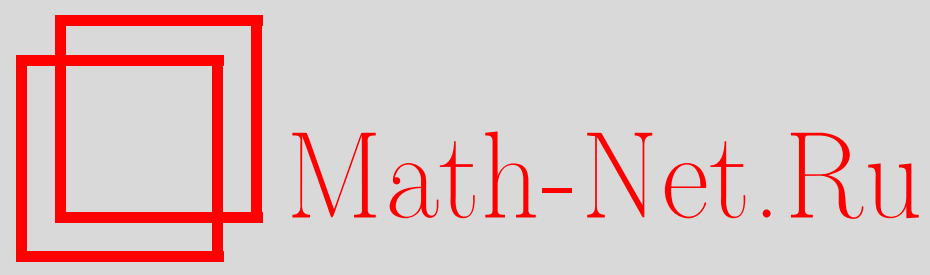

П. де ля Арп, М. Бушер, Свободные произведения с объединением и $\mathrm{HNN}$-расширения равномерно экспоненциального роста, Матем. заметки, 2000, том 67, выпуск 6, 811-815

DOI: https://doi.org/10.4213/mzm899

Использование Общероссийского математического портала Math-Net.Ru подразумевает, что вы прочитали и согласны с пользовательским соглашением http://www.mathnet.ru/rus/agreement

Параметры загрузки:

IP : 54.92 .164 .108

26 апреля 2023 г., 18:36:37

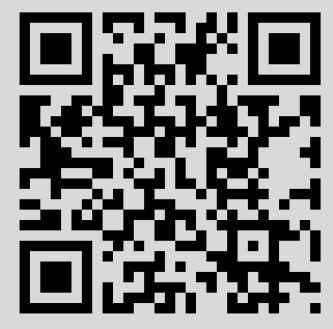




\title{
СВОБОДНЫЕ ПРОИЗВЕДЕНИЯ \\ С ОБЪЕДИНЕНИЕМ И НNN-РАСШИРЕНИЯ РАВНОМЕРНО ЭКСПОНЕНЦИАЛЬНОГО РОСТА
}

\author{
П. де ля Арп, М. Бушер
}

\begin{abstract}
Доказано, что группы, являющиеся свободным произведением с объединением или HNN-расширением, обладают, за редким исключением, равномерно экспоненциальньмм ростом.

Библиограффия: 8 названий.
\end{abstract}

Пусть $\Gamma$ - группа с конечньм множеством порождающих $S$. Тогда определены: $c л о$ варная длина $\ell_{S}: \Gamma \rightarrow \mathbb{N}$, где $\ell_{S}(\gamma)$ - наименьшее целое число $n$ такое, что найдутся элементы $s_{1}, \ldots, s_{n} \in S \cup S^{-1}$, удовлетворяющие соотношению $\gamma=s_{1} \cdots s_{n}$; иия роста $\beta_{S}: \mathbb{N} \rightarrow \mathbb{N}$, где $\beta_{S}(k)$ - число элементов $\gamma \in \Gamma$ длины $\ell_{S}(\gamma) \leqslant k$; а также показатель әкспоненииального роста

$$
\omega(\Gamma, S)=\lim _{k \rightarrow \infty} \sqrt[k]{\beta_{S}(k)}
$$

(предел существует ввиду полумультипликативности функции роста: $\beta_{S}\left(k_{1}+k_{2}\right) \leqslant$ $\left.\beta_{S}\left(k_{1}\right) \beta\left(k_{2}\right)\right)$. Легко проверить, что $\omega(\Gamma, S)>1$ в том и только том случае, если $\omega\left(\Gamma, S^{\prime}\right)>1$ для произвольного конечного множества $S^{\prime}$ порождающих Г. Определим также показатель

$$
\omega(\Gamma)=\inf \{\omega(\Gamma, S)\},
$$

где инфинум берется по всем $S$ - конечным множествам порождающих группы Г. Группа имеет экспоненииальный рост, если $\omega(\Gamma, S)>1$ для некоторого (а значит, и для любого) конечного порождающего множества $S$, и имеет равномерно әкспоненциальны й рост, если $\omega(\Gamma)>1$. Громовым был поставлен вопрос о существовании конечно порожденной грушы экспоненциального, но не равномерно экспоненциального роста [1, замечание 5.12], однако вопрос до сих пор остается открытым [2] (см. [3], [4, гл. VII], где эта проблема обсуждается более подробно).

Цель заметки - показать, что при некоторых естественных условиях свободное произведение с объединением $A *_{C} B$, где $C$ - общая подгруппа в $A$ и $B$, и HNN-pacширение $G *_{H}^{\theta}=\left\langle G, t \mid \theta(h)=t h t^{-1} \forall h \in H\right\rangle$, где $H$ - подгрупша в $G$ и $\theta$ - изоморфизм между $H$ и ее образом в $G$, имеют равномерно экспоненциальный рост. Точнее, мы доказываем следующий результат, используя при этом стандартное обозначение $[A: C] \in\{1,2, \ldots, \infty\}$ для индекса подгруппы $C$ в группе $A$. 
Теорема. і) Пусть $A, B$ - две конечно порохденные группь, и предполохим, что $C$ - группа, содержащаяся одновременно в $A$ и В. Предположим, кроме того, что выполнено условие

$$
([A: C]-1)([B: C]-1) \geqslant 2 .
$$

Тогда справедлива оченка

$$
\omega\left(A *_{C} B\right) \geqslant \sqrt[4]{2}
$$

В частности, свободное произведение с объединением $A *_{C} B$ имеет равномерно әкспоненциальный рост.

ii) Пусть $H, K$ - изоморфные подгруппы конечно порохденной группы $G$ и

$$
\theta: H \rightarrow K
$$

- изоморфизм между ними. Более того, предположим, что $[G: H]+[G: K] \geqslant 3$. Тогда справедлива оченка

$$
\omega\left(G *_{H}^{\theta}\right) \geqslant \sqrt[4]{2}
$$

В частности, HNN-расширение $G *_{H}^{\theta}$ обладает равномерно әкспоненциальным ростом.

Известно, что групшы $\Gamma=A *_{C} B$ и $\Delta=G *_{H}^{\theta}$ действуют на деревьях $X$ и $Y$ соответственно, которые определяются следующим образом.

i) Множество вершин $X^{0}$ графа $X$ является непересекаюшимся объединением фактормножеств $\Gamma / A$ и $\Gamma / B$. Множество ребер состоит из дизьюнкного объединения $X^{1}=$ $X_{+}^{1} \sqcup \overline{X_{+}^{1}}$ с положительной частью $X_{+}^{1}=\Gamma / C$, причем ребро $\gamma C \in X_{+}^{1}$ имеет начало $\gamma A$ и конец $\gamma B$ (см. [5, № I.4.1]). Дерево $X$ является двудольным, вершины из $\Gamma / A$ (соответственно из $\Gamma / B)$ имеют степень $[A: C]$ (соответственно $[B: C])$, и всякий элемент $\gamma \in \Gamma$ определяет автоморфизм $X$, который является четным, т.е. комбинаторное расстояние $d(x, \gamma(x))$ является четным числом для любой вершины $x \in X^{0}$.

ii) Множеством вершин графа $Y$ служит $Y^{0}=\Gamma / G$. Множество ребер $Y^{1}=Y_{+}^{1} \sqcup \overline{Y_{+}^{1}}$ обладает положительной частью $Y_{+}^{1}=\Gamma / H$, и ребро $\delta H \in Y_{+}^{1}$ имеет начало $\delta G$ и конец $\delta t G$ (см. [5, № I.5.3]). Заметим, что $Y$ является регулярньп деревом степени

$$
[G: H]+[G: K]
$$

СЛЕДСТВИЕ. Пусть $G$ - конечно порожденная группа. Если у нее существует конечное множество образующих $S$ такое, что $\omega(G, S)<\sqrt[4]{2}$, то $G$ не является ни свободным произведением с обвединением вида $A *_{C} B$, где

$$
([A: C]-1)([B: C]-1) \geqslant 2
$$

ни HNN-расширением вида $G *_{H}^{\theta}$, дде $[G: H]+[G: \theta(H)] \geqslant 3$.

Сформулированная выше теорема является непосредственным следствием следующего утверждения. 
ПРЕДЛОЖЕНИЕ. Пусть $X$ - дерево и конечно порожденная группа $Г$ действует автоморфизмами на $X$. Предположим, что либо Г транзитивна на $X^{0}$, либо граф $X$ двудолен, Г действует четными автоморфизмами на $X$ и имеет в точности две орбиты на $X^{0}$. Кроме того, предположим, что граф $X$ бесконечен $u$ не изоморфен прямой. Тогда $\omega(\Gamma, S) \geqslant \sqrt[4]{2}$ для произвольного конечного множества $S$ порождающих $\Gamma$.

Напомним, что автоморфизм $s$ графа $X$ является гиперболическим, если у него нет неподвижных вершин и нет неподвижных геометрических ребер. Для такого $s \in \operatorname{Aut}(X)$ найдется геодезическая $L_{s}$ в $X$, назьваемая осью $s$, с множеством вершин

$$
\left\{x \in X^{0} \mid d(x, s(x))=\min _{y \in X^{0}} d(y, s(y))\right\} .
$$

Лемма. Рассмотрим два гиперболических автоморфизма $s, t$ дерева $X$. Пусть $\Gamma_{0}$ - группа, ими порохденная, и пусть $S_{0}=\{s, t\} . \operatorname{Ecлu} L_{s} \neq L_{t}, \operatorname{mo} \omega\left(\Gamma_{0}, S_{0}\right) \geqslant 2$.

ДокАЗАТЕЛьство. Занумеруем вершины $\left(x_{n}\right)_{n \in \mathbb{Z}}$ оси $L_{s}$ и $\left(y_{n}\right)_{n \in \mathbb{Z}}$ оси $L_{t}$ таким образом, чтобы выполнялись следуюшие условия:

i) $d\left(x_{m}, x_{n}\right)=d\left(y_{m}, y_{n}\right)=|m-n|$, где $m, n \in \mathbb{Z}$;

ii) если $L_{s}$ и $L_{t}$ не пересекаются, то геодезический отрезок в $X$, соединяющий $L_{s}$ с $L_{t}$, имеет $x_{0}$ и $y_{0}$ своими концами;

iii) если же $L_{s}$ и $L_{t}$ пересекаются, то $x_{0}=y_{0}$ и $x_{m} \neq y_{n}$ при $m, n>0$.

Заменив в случае необходимости $s$ на $s^{-1}$ и $t$ на $t^{-1}$, мы можем, кроме того, предположить, что существуют числа $a, b \geqslant 1$ такие, что $s\left(x_{n}\right)=x_{n+a}$ и $t\left(y_{n}\right)=y_{n+b}$ при любом $n \in \mathbb{Z}$. Пусть $P$ (соответственно $Q$ ) - подмножество в $X^{0}$, состоящее из вершин $z$ таких, что $d\left(z, x_{1}\right)=d\left(z, x_{0}\right)-1$ (соответственно $\left.d\left(z, y_{1}\right)=d\left(z, y_{0}\right)-1\right)$. Тогда $P \cap Q=\varnothing$ и $s^{k}(P \cup Q) \subset P, t^{k}(P \cup Q) \subset Q$, если $k \geqslant 1$. С помощью стандартого утверждения, называемого пинг-понг-леммой (см., например, [4, предложение VII.2]), получаем, что $S_{0}$ порождает свободную полугрупу $M_{0}$ с двумя образующими. Понятие экспоненциального роста аналогичным образом можно расширить и на полугрупшы, тогда $\omega\left(M_{0}, S_{0}\right)=2$. Следовательно,

$$
\omega\left(\Gamma_{0}, S_{0}\right) \geqslant \omega\left(M_{0}, S_{0}\right)=2
$$

и лемма доказана.

ДоКАЗАТЕЛЬСТВо ПРЕДЛОЖЕНИЯ. ПредПоложим вначале, что $S$ содержит элемент $s$, являющийся гиперболическим на $X$ с осью $L_{s}$. Если бы имели место соотношения $u\left(L_{s}\right)=L_{s}, u \in S$, то также имели бы место соотношения $\gamma\left(L_{s}\right)=L_{s}, \gamma \in \Gamma$, а значит, и соотношения $L_{s}=X$, так как $\Gamma$ вопреки наложенным условиям имеет не более двух орбит в $X^{0}$. Следовательно, существует $t \in S$ такое, что $t\left(L_{s}\right) \neq L_{s}$. Если $S_{0}=\left\{s, t s t^{-1}\right\}$ и $\Gamma_{0}$ является подгруппой $\Gamma$, порожденной $S_{0}$, то $\omega\left(\Gamma_{0}, S_{0}\right) \geqslant 2$ по доказанной лемме.

Пусть $B(k)$ обозначает шар радиуса $k$ с центром $e$ в $\Gamma$ по отношению к словарной длине $\ell_{S}$, так что $S_{0} \subset B(3)$. Заметим, что $\omega(\Gamma, B(k))=\omega(\Gamma, S)^{k}$ при $k \geqslant 1$. Поэтому выполняется равенство

$$
\omega(\Gamma, S)^{3}=\omega(\Gamma, B(3)) \geqslant \omega\left(\Gamma_{0}, S_{0}\right) \geqslant 2,
$$

что и заканчивает рассуждение в рассмотренном случае. 
Предположим теперь, что любой автоморфизм $u \in S$ имеет некоторую фиксированную вершину в $X^{0}$. Тогда найдется элемент $s \in S \cup S^{2}$, являющийся гиперболическим на $X$, ввиду [5, № I.6.5]. Те же аргументы, что и выше, показывают, что в этом случае

$$
\omega(\Gamma, S)^{4}=\omega(\Gamma, B(4)) \geqslant 2
$$

и утверждение полностью доказано.

ЗАмечаниЕ 1 . Для свободного произведения $\Gamma=A * B$ двух груп, удовлетворяющих условию $(|A|-1)(|B|-1) \geqslant 2$, оценка может быть улучшена до $\omega(\Gamma) \geqslant \sqrt{2}$. Эта оценка является строгой, так как если $A=\{1, \alpha\}, B=\left\{1, \beta, \beta^{2}\right\}$ и $S=\{\alpha, \beta\}$, то, полагая $\sigma_{S}(k)=\beta_{S}(k)-\beta_{S}(k-1)$, мы получаем соотношение $\sigma_{S}(k+2)=2 \sigma_{S}(k)$, выполненное для любого $k \geqslant 1$, так что

$$
\omega(A * B, S)=\lim _{k \rightarrow \infty} \frac{\sigma_{S}(k+2)}{\sigma_{S}(k+1)}=2 \lim _{k \rightarrow \infty} \frac{\sigma_{S}(k)}{\sigma_{S}(k+1)}=\frac{2}{\omega(A * B, S)}
$$

и, следовательно, $\omega(A * B, S)=\sqrt{2}$.

Для всех примеров групп, удовлетворяющих условиям нашей теоремы, для которых мы смогли провести точные вычисления, показатель равномерно экспоненциального роста также удовлетворяет оценке $\omega(\cdot) \geqslant \sqrt{2}$.

ЗАмечание 2 . Случай групш вида $\Gamma=A *_{C} B$ с условием $([A: C]-1)([B: C]-1)=2$ не является тривиальным.

Существует пример, принадлежащий Брайдсону, в котором $C \approx \mathbb{Z}^{2}$ и $[A: C]=$ $[B: C]=2$ и для которого $C$ не является, а $\Gamma=A *_{C} B$ является группой экспоненциального роста (см. [4, дополнение VII.10]). В примере Брайдсона Г обладает подгруппой индекса 2 , изоморфной полупрямому произведению вида $\mathbb{Z}^{2} \rtimes_{\theta} \mathbb{Z}, \theta=\left(\begin{array}{cc}0 & 1 \\ -1 & 3\end{array}\right)$, так что Г обладает равномерно экспоненциальньм ростом (конечно порожденная группа и подгруппа конечного индекса одновременно являются или не являются группами с равномерно экспоненциальным ростом, что следует из [6, предложение 3.3$]$ ).

Если $C=1$ и группы $A, B$ одновременно имеют порядок 2 , то $A * B$ является бесконечной диэдральной группой и поэтому имеет линейньй рост.

ЗАмечаниЕ 3 . Аналогично, если $H=K=G$, то HNN-расширение $G *_{H}^{\theta}$ на самом деле есть полупрямоепроизведение $\Delta=G \rtimes_{\theta} \mathbb{Z}$. Если $G$ не обладает экспоненциальным ростом, то $\Delta$ является или не является группой экспоненциального роста в зависимости от свойств $\theta$. Например, для $G=\mathbb{Z}^{d}$ и $\theta \in G L(d, \mathbb{Z})=\operatorname{Aut}\left(\mathbb{Z}^{d}\right)$ группа $\Delta$ обладает полиномиальным ростом, если автоморфизм $\theta$ является унипотентным, и обладает равномерно экспоненциальным ростом, если $\theta$ имеет вид, рассмотренный ниже в примере.

ПримеР. Пусть $d$ - целое число, $d \geqslant 2$, и пусть $\theta \in G L(d, \mathbb{Z})$ - матрица с собственным значением $\lambda$ таким, что $|\lambda|>1$. Тогда соответствующее полупрямое произведение $\Delta=\mathbb{Z}^{d} \rtimes_{\theta} \mathbb{Z}$ имеет равномерно экспоненциальньй рост.

В частности, группа из рассмотренного ниже примера имеет экспоненциальньй рост. Этот результат получен еще в 1955 году Шварцем [7].

ДокАЗАТЕльство. Пусть $S$ - порождающее множество группы $\Delta$. Обозначим через $\pi: \Delta \rightarrow \mathbb{Z}$ каноническую проекцию. Тогда найдется элемент $s \in S$ такой, что $\pi(s)=m \neq 0$. Изменив в случае необходимости $s$ на $s^{-1}$, можно предположить, что $m \geqslant 1$. Поскольку группа $\Delta$ не абелева, существует элемент $v \in S$ такой, что $s v \neq v s$. 
Положим $u=s v s^{-1} v^{-1}$. Так как $u \in \mathbb{Z}^{d}$, можно отождествить $s u s^{-1}$ с $\theta^{m}(u)$. Положим $S_{0}=\{s, u\}$ и пусть $\Delta_{0}-$ подгруппа в $\Delta$, порожденная множеством $S_{0}$. Так как $S_{0}$ находится внутри шара радиуса 4 с центром $е$ в группе $\Delta$ по отношению к словарной длине $\ell_{S}$, то $\omega(\Delta, S)^{4} \geqslant \omega\left(\Delta_{0}, S_{0}\right)$ и, следовательно, достаточно показать, что $\omega\left(\Delta_{0}, S_{0}\right) \geqslant c$ для некоторой константы $c>1$, не зависящей от выбора $S$.

Обозначим через $L$ линейную форму на $\mathbb{R}^{d}$ такую, что $L \circ \theta=\lambda L$. Имеем $L(u) \neq 0$, так как $u \in \mathbb{Z}^{d}$ и $\lambda \notin \mathbb{Q}$. Мы будем предполагать, что $\lambda$ является вещественным, а случай двух комплексно сопряженных значений $\lambda, \bar{\lambda}$ оставим читателю в качестве упражнения. Пусть $n \geqslant 1$ таково, что $|\lambda|^{n} \geqslant 2$; это число зависит от $\theta$, но не от $S$. Заметим, что $|\lambda|^{m n} \geqslant 2$. При $k \geqslant 1$ и $\varepsilon_{1}, \ldots, \varepsilon_{k} \in\{0,1\}$ имеем

$$
L\left(\varepsilon_{1} u+\varepsilon_{2} s^{n} u s^{-n}+\cdots+\varepsilon_{k} s^{n(k-1)} u s^{-n(k-1)}\right)=\left(\sum_{j=0}^{k-1} \varepsilon_{j} \lambda^{j m n}\right) L(u),
$$

причем числа

$$
\sum_{j=0}^{k-1} \varepsilon_{j}\left(\lambda^{m n}\right)^{j}
$$

различны. С другой стороны, в группе $\Delta=\mathbb{Z}^{d} \rtimes_{\theta} \mathbb{Z}$ выполнено соотношение

$\left(\varepsilon_{1} u, m n\right)\left(\varepsilon_{2} u, m n\right) \cdots\left(\varepsilon_{k} u, m n\right)=\left(\varepsilon_{1} u+\varepsilon_{2} s^{n} u s^{-n}+\cdots+\varepsilon_{k} s^{n(k-1)} u s^{-n(k-1)}, k m n\right)$,

так что $\ell_{S_{0}}$ - словарная длина этого элемента - не превосходит $(n+1) k$. Следовательно, функция роста пары $\left(\Delta_{0}, S_{0}\right)$ удовлетворяет неравенству $\beta_{S_{0}}((n+1) k) \geqslant 2^{k}$, и окончательно получаем $\omega\left(\Delta_{0}, S_{0}\right) \geqslant 2^{1 /(n+1)}$ (это рассуждение в значительной степени аналогично доказательству леммы 6.2 из [8]).

Авторы выражают благодарность Р. И. Григорчуку за его замечания к первой версии этой заметки и Национальному научному фонду Швейцарии, которьй поддержал его визит в Женеву.

\section{СПИСОК ЦИТИРОВАННОЙ ЛИТЕРАТУРЫ}

[1] Gromov M., Lafontaine J., Pansu P. Structures métriques pour les variétés riemanniennes: Cedic/F. Nathan, 1981.

[2] Gromov M. Metric Structures for Riemannian and non-Riemannian Spaces (with appendices by M. Katz, P. Pansu, and S. Semmes): Birkhäuser, 1999.

[3] Grigorchuk R. I., de la Harpe P. Limit behaviour of exponential growth rates for finitely generated groups // Preprint at http://www .unige.ch/math/biblio/preprint/pp98.html: Univ. of Geneva, 1999.

[4] de la Harpe P. Topics in Geometric Group Theory: Univ. of Chicago Press (to appear).

[5] Serre J.-P. Arbres, amalgames, $S L_{2}$. Astérisque. V. 46: Soc. Math. France, 1977.

[6] Shalen P. B., Wagreich P. Growth rates, $\mathbb{Z}_{p}$-homology, and volumes of hyperbolic 3-manifolds // Trans. Amer. Math. Soc. 1992. V. 331. P. 895-917.

[7] Шварц А. С. Объемный инвариант накрытий // Докл. АН СССР. 1955. Т. 105. С. 32-34.

[8] Tits J. Groupes à croissance polynomiale // Séminaire Bourbaki 1980/81. Lecture Notes in Math. V. 901: Springer, 1981. P. 176-188.

(П. де ля Арп) Отделение математики, Женевский университет, Цюрих (М. Бушер) Отделение математики, ЕТН-Центр 